\title{
Autonomic dysfunction associated with Type 1 diabetes: a role for fitness?
}

\author{
Ryan McGinn · Glen P. Kenny
}

Received: 7 November 2014/Accepted: 7 November 2014/Published online: 15 November 2014

(C) Springer-Verlag Berlin Heidelberg 2014

A robust and highly responsive autonomic nervous system (ANS) is an invaluable component to human health. The ANS innervates and governs most human organ systems via its two subdivisions, the sympathetic and parasympathetic nerves. For example, patients with differing levels of ANS dysfunction can present with impairments in blood pressure regulation, control of heart rate, and thermoregulation to name a few [6]. While changes in autonomic control have been linked to several chronic diseases and disorders, the large fluctuations in blood glucose levels associated with Type 1 diabetes mellitus (T1DM) tend to place these patients at particularly greater risk [3]. The impairment in ANS function associated with T1DM has been noted in numerous studies, even in those patients who appear to be otherwise "healthy" (i.e., no diagnosed retinopathy, nephropathy, or clinical neuropathy) [11, 12]. A recently published study by Limberg et al. [8] not only eloquently highlights how ANS function is impaired in patients with T1DM at rest, but also how factors as simple as glycemic management can exacerbate impairments in autonomic control. Specifically, these findings were evidenced by marked reductions in heart rate variability, spontaneous cardiac baroreflex sensitivity, and T-wave amplitude along with prolonged QT intervals during and following an acute hypoglycemic episode [8]. Considering that $\sim 82 \%$ of patients with T1DM report at least 1

R. McGinn · G. P. Kenny ( $₫)$

Human and Environmental Physiology Research Unit, School of Human Kinetics, University of Ottawa, Ottawa, ON K1N 6N5,

Canada

e-mail: gkenny@uottawa.ca

G. P. Kenny

125 University Private, Room 367, Montpetit Hall, Ottawa,

ON K1N 6N5, Canada hypoglycemic event each month [4], with greater frequencies reported during physically demanding tasks or physical exercise [14], implementing interventions which can delay and/or reverse ANS dysfunction in these patients is paramount.

Along with individualized diet and insulin regimens prescribed for patients with T1DM, exercise has long been considered a key component to diabetes management [2]. Along with the improvements associated with cardiorespiratory health and reductions in the risk for chronic disease, exercise training has clearly been shown to improve ANS function [6]. In fact, the physiological adjustments involving the ANS are believed to play a major role in the systemic protective and therapeutic effects of exercise training [7]. Furthermore, exercise training has been shown to augment baroreflex function and even blunts the agerelated reductions in baroreflex sensitivity $[7,10]$. To date, most of these studies have focussed on "healthy" populations; however, the effects and benefits of chronic exercise training in population groups with impaired ANS function (i.e., patients with T1DM) remain largely unknown. Specifically in the study by Limberg et al. [8], fitness of the groups with and without T1DM was not controlled, albeit the authors excluded any individuals who reportedly engaged in regular physical activity programs. Therefore, considering the strong evidence for exercise-induced improvements in ANS function [6], future studies should include separate patient groups with T1DM based on fitness to examine the potential impact of chronic exercise training on blunting and/or reversing impairments in ANS function.

Despite the insurmountable evidence for the health benefits associated with chronic exercise training, there remain certain risks related to acute exercise bouts. A recent report by Carter and colleagues [1] revealed that 
T1DM may cause changes in the body's normal response to heat exposure. When combined with the recent observations by Limberg et al. [8] of impaired autonomic control, this may imply that individuals with T1DM may be at a greater risk of suffering from a heat-related injury or death during challenges to human heat balance associated with elevations in environmental heat load, metabolic rate during physical activity, or a combination of the two. Specifically, the study by Carter et al. [1] showed that otherwise healthy individuals with T1DM exhibited a reduction in sweating during prolonged incremental exercise (i.e., three 30-min exercise bouts performed at increasing fixed rates of heat production of 200, 250, and $300 \mathrm{~W} \mathrm{~m}^{-2}$ or 45,55 , and $65 \%$ of maximal oxygen consumption, respectively) in the heat compared to their counterparts without T1DM (matched for fitness, age, and anthropometrics). Evaporation of sweat represents the primary avenue of heat loss during exercise in the heat. Consequently, this reduction in sweating for individuals with T1DM was associated with a greater increase (by $\sim 0.5^{\circ} \mathrm{C}$ ) in rectal temperature, thereby indicating greater heat storage during exercise. Altogether, these data suggest that T1DM is associated with an invariably greater risk for heat-related injury, especially under conditions which may reduce sweating efficiency (i.e., the amount of sweat evaporated and lost as heat compared to the amount of sweat produced) such as exercise performed in highly humid conditions or when wearing clothing as insulation [1].

Heart rate variability (HRV) has been identified as a window over the ANS and the level at which it is functioning. In healthy young adults, Flouris et al. [5] found that marked exercise-induced hyperthermia (i.e., core body temperature $\geq 39.5^{\circ} \mathrm{C}$ ) had a significant effect on ANS control as assessed by HRV which was restored only when core body temperature returned to pre-exercise baseline resting levels. Whether or not this pattern of response is exacerbated in individuals with T1DM or occurs at a lower level of heat stress remains to be elucidated. However, given that Carter et al. [1] showed the suppression of the sweating response to be more pronounced with increasing levels of heat stress relative to their healthy counterparts would support this possibility. Further, despite this increased level of heat strain during exercise, and therefore thermal drive, a follow-up study with the same participants demonstrated that the individuals with T1DM did not exhibit a compensatory increase in the rate of heat loss during 60 min of recovery [9]. Rather, it was reported that the individuals with T1DM sustained a marked impairment in heat loss that was paralleled by a concomitant reduction in baroreflex sensitivity throughout the $60 \mathrm{~min}$ recovery period compared to their healthy counterparts. Taken together, these findings demonstrate that T1DM is associated with a progressive decrease in autonomic control leading to impairments in thermoregulatory and cardiovascular function. Dysfunction in these systems can result in significant reductions in the body's physiological capacity to dissipate heat during exercise and thereby the ability to regulate core body temperature, especially in the heat. If combined with the disruptions associated with an acute hypoglycemic episode in patients with T1DM [8], the risk for autonomic failure and/or fatal cardiac arrhythmia may be augmented or additive.

Evidence clearly demonstrates that chronic exercise training is beneficial for improving ANS control in healthy individuals [6], which is paralleled by increased cardiovascular and thermoregulatory function. However, the impact of such exercise training on individuals with T1DM (i.e., patients with impaired ANS function even at rest) remains to be elucidated. Furthermore, the acute risks of a single exercise bout cannot be overlooked, especially from a glucoregulatory perspective. Consequently, future research should be directed at determining the most appropriate exercise paradigm for patients with T1DM yielding the lowest risk and greatest reward, while also considering the influence of exercise-induced increases in metabolic heat production. As is clearly demonstrated in the study by Limberg and colleagues [8], patients with T1DM exhibit exacerbated autonomic dysregulation during a period of hypoglycemia which may be precipitated by a bout of exercise. Specifically, recent work has determined that aerobic exercise leads to the acute and pronounced reductions of glycemia in patients with T1DM [14]. However, it is important to consider that when resistance and aerobic exercise are performed in sequence, a normalization of glycemic control is observed [13]. While ANS function was not evaluated in these studies, it is possible that the normalization of glycemia (by performing resistance exercise followed by aerobic exercise) may be associated with less pronounced impairments of autonomic regulation during postexercise recovery.

In summary, the management of T1DM is reliant upon an integrated regimen of diet, insulin dosing, and exercise. However, much remains unknown regarding the effects and benefits of chronic exercise training on T1DM. Specifically, it remains unclear to what extent a role for fitness exists in determining the level of autonomic dysfunction in patients with T1DM and in turn, its effects on cardiovascular and thermoregulatory function especially during exercise-induced heat stress. Considering the potentially catastrophic effects of impaired ANS function on one's health, future studies should be aimed first to determine if and how increasing fitness can dampen and/or reverse the impairments associated with T1DM, and then to determine the most efficacious manner of performing the necessary interventions. 


\section{References}

1. Carter MR, McGinn R, Barrera-Ramirez J, Sigal RJ, Kenny GP (2014) Impairments in local heat loss in Type 1 diabetes during exercise in the heat. Med Sci Sports Exerc. doi:10.1249/MSS. 0000000000000350

2. Chimen M, Kennedy A, Nirantharakumar K, Pang TT, Andrews R, Narendran P (2012) What are the health benefits of physical activity in type1 diabetes mellitus? A literature review. Diabetologia 55:542-551

3. Davis SN, Mann S, Briscoe VJ, Ertl AC, Tate DB (2009) Effects of intensive therapy and antecedent hypoglycemia on counterregulatory responses to hypoglycemia in type 2 diabetes. Diabetes 58:701-709

4. Donnelly LA, Morris AD, Frier BM, Ellis JD, Donnan PT, Durrant R, Band MM, Reekie G, Leese GP (2005) Frequency and predictors of hypoglycaemia in Type 1 and insulin-treated Type 2 diabetes: a population-based study. Diabet Med 22:749-755

5. Flouris AD, Bravi A, Wright-Beatty HE, Green G, Seely AJ, Kenny GP (2014) Heart rate variability during exertional heat stress: effects of heat production and treatment. Eur J Appl Physiol 114:785-792

6. Fu Q, Levine BD (2013) Exercise and the autonomic nervous system. Handb Clin Neurol 117:147-160

7. Joyner MJ, Green DJ (2009) Exercise protects the cardiovascular system: effects beyond traditional risk factors. J Physiol 587:5551-5558

8. Limberg JK, Farni KE, Taylor JL, Dube S, Basu A, Basu R, Wehrwein EA, Joyner MJ (2014) Autonomic control during acute hypoglycemia in type1 diabetes mellitus. Clin Auton Res. doi:10. 1007/s10286-014-0253-y

9. McGinn R, Carter MR, Barrera-Ramirez J, Sigal RJ, Flouris AD, Kenny GP (2014) Does Type 1 Diabetes alter postexercise thermoregulatory and cardiovascular function in young adults? Scand J Med Sci Sports doi: SJMSS-SA-323-314

10. Monahan KD, Dinenno FA, Tanaka H, Clevenger CM, DeSouza CA, Seals DR (2000) Regular aerobic exercise modulates ageassociated declines in cardiovagal baroreflex sensitivity in healthy men. J Physiol 529(Pt 1):263-271

11. Weston PJ, James MA, Panerai RB, McNally PG, Potter JF, Thurston H (1998) Evidence of defective cardiovascular regulation in insulin-dependent diabetic patients without clinical autonomic dysfunction. Diabetes Res Clin Pract 42:141-148

12. Weston PJ, Panerai RB, McCullough A, McNally PG, James MA, Potter JF, Thurston H, Swales JD (1996) Assessment of baroreceptor-cardiac reflex sensitivity using time domain analysis in patients with IDDM and the relation to left ventricular mass index. Diabetologia 39:1385-1391

13. Yardley JE, Kenny GP, Perkins BA, Riddell MC, Malcolm J, Boulay P, Khandwala F, Sigal RJ (2012) Effects of performing resistance exercise before versus after aerobic exercise on glycemia in type 1 diabetes. Diabetes Care 35:669-675

14. Yardley JE, Kenny GP, Perkins BA, Riddell MC, Malcolm JS, Sigal RJ (2010) Greater fluctuations in blood glucose seen both during and after aerobic exercise as compared to resistance exercise or no exercise in type 1 diabetes: a study using continuous glucose monitoring. Appl Physiol Nutr Metab 35:S112 